\title{
Medizinisches Denken bei den Byzantinern
}

La pensée médicale chez les Byzantins

Medical Reflexions among Byzantines

\section{Armin Hohlweg}

\section{(2) OpenEdition}

Journals

Édition électronique

URL : http://journals.openedition.org/etudesbalkaniques/352

ISSN : 2102-5525

\section{Éditeur}

Association Pierre Belon

\section{Édition imprimée}

Date de publication : 1 avril 1994

Pagination : 81-101

ISBN : 2-910860-01-9

ISSN : $1260-2116$

\section{Référence électronique}

Armin Hohlweg, « Medizinisches Denken bei den Byzantinern », Études balkaniques [En ligne], 1 | 1994, mis en ligne le 08 avril 2009, consulté le 24 avril 2019. URL : http://journals.openedition.org/ etudesbalkaniques/352

Ce document a été généré automatiquement le 24 avril 2019

Tous droits réservés 


\title{
Medizinisches Denken bei den
} Byzantinern

\author{
La pensée médicale chez les Byzantins \\ Medical Reflexions among Byzantines
}

\section{Armin Hohlweg}

\section{RÉSUMÉS}

Les médecins byzantins étaient les héritiers de la médecine d'Hippocrate, en ce sens qu'ils ne se contentaient pas de spéculations ou de théorisations mais s'astreignaient à expliquer les phénomènes de façon rationnelle et à développer une pratique satisfaisante. Toutefois le christianisme infléchit la médecine ancienne. L'attachement à l'être humain, créé à l'image de Dieu, motive les soins médicaux d'une nouvelle façon. Le christianisme tente aussi de repousser les pratiques superstitieuses qui éloignent de la foi orthodoxe et contribue à clarifier les limites du champ médical, sans que toutefois on puisse nier l'aspect psychosomatique de pratiques « hiatrosophiques » inextricables. Il faut se rendre compte que les médecins byzantins n'étaient pas de simples épigones serviles des Anciens, comme le montre l'auteur en évoquant des écrits critiques, à partir du XIe siècle, qui introduisaient pour un public composé de savants pas tous du champ médical proprement dit et s'intéressait à des mise en question même de la théorie des humeurs. Enfin, la médecine byzantine tardive a sans aucun doute influencé le renouveau médical en Occident.

Byzantine medical doctors were the heirs of Hippocrates since they did not indulge only in speculations or unquestioned theories but compelled themselves to rational explanations of phenomena and rewarding practices. However Christianity modified Ancient medicine. Viewing 
human beings as made in God's image re-motivated medical care. Christianity tried to suppress superstitious practices that contradicted Orthodoxy and thus contributed to define clearly the realm of rational medicine, though some psychosomatic aspect of "hiatropsophy" could not be neither neglected nor rejected. One has to accept that Byzantine medical doctors were not sterile imitators of the Ancient times. Hohlweg makes his point quoting critical texts from the 11th century onward that presented to a readership made up of scholars from various fields and not medical doctors only that was interested in questioning the theory of four humors. Finally, the late Byzantine medical art influenced with no possible doubt the Western medical Renaissance.

\section{AUTEUR}

\section{ARMIN HOHLWEG}

Professeur - Institut de la philologie byzantine et néo-hellénique et de l'histoire de l'art byzantin, Université de Munich 